\title{
Stylization and Trajectory Modelling of Short and Long Term Speech Prosody Variations
}

\author{
Nicolas Obin ${ }^{1,2}$ \\ Anne Lacheret ${ }^{2}$, Xavier Rodet ${ }^{1}$ \\ ${ }^{1}$ Analysis-Synthesis Team, IRCAM, Paris, France \\ ${ }^{2}$ Modyco Lab., University of Paris Ouest - La Défense, Nanterre, France \\ nobin@ircam.fr, anne.lachereteu-paris10.fr, rodeteircam.fr
}

\begin{abstract}
In this paper, a unified trajectory model based on the stylization and the modelling of $f_{0}$ variations simultaneously over various temporal domains is proposed ${ }^{1}$ The syllable is used as the minimal temporal domain for the description of speech prosody, and short-term and long-term $f_{0}$ variations are stylized and modelled simultaneously over various temporal domains. During the training, a context-dependent model is estimated according to the joint stylized $f_{0}$ contours over the syllable and a set of long-term temporal domains. During the synthesis, $f_{0}$ variations are determined using the long-term variations as trajectory constraints. In a subjective evaluation in speech synthesis, the stylization and trajectory modelling of short and long term speech prosody variations is shown to consistently model speech prosody and to outperform the conventional short-term modelling.
\end{abstract}

Index Terms: speech prosody, stylization, trajectory model, speech synthesis.

\section{Introduction}

In parallel to the development of high-quality speech synthesis systems [1], the modelling of speech prosody has raised as a major concern to improve the naturalness, the liveliness, and the variety of the synthetic speech. Speech prosody is generally described as the co-occurrence of acoustic gestures occurring simultaneously over different temporal domains [2, 3] and associated to different communicative functions (linguistic, expressive). A high-quality modelling of speech prosody is desirable for natural and expressive speech synthesis and adequate modelling of speaking style, and a prerequisite in real multi-media applications (e.g., avatars, story telling, dialogue systems, numeric arts).

A variety of methods has been proposed to model speech prosody variations $\left(f_{0}[4]\right.$, temporal structure [5]), and local and global variations [6, 7]. However, conventional methods usually models short-term variations of speech prosody (frame-based, or instantaneous variations), while long-term variations of speech prosody are not explicitly considered. Recent studies have been proposed to integrate long-term variations into HMM modelling, either for the modelling of $f_{0}$ variations [8, 9], or with extension to state-duration

\footnotetext{
${ }^{1}$ This study was partially funded by "La Fondation Des Treilles", and supported by ANR Rhapsodie 07 Corp-030-01; reference prosody corpus of spoken French; French National Agency of research; 20082012.
}

modelling [10]. However, the proposed methods remain a mixed model, i.e. the conventional model is used to model the instantaneous variations of $f_{0}$, while stylization of long-term variations are used as trajectory constraints only. In particular, the instantaneous variations remain the minimal and target temporal domain for the modelling of speech prosody.

In this paper, a unified trajectory model based on the stylization and the joint modelling of $f_{0}$ variations over various temporal domains is proposed. In the proposed approach, the syllable is used as the minimal temporal domain for the description of speech prosody, and $f_{0}$ variations are stylized and modelled simultaneously over various temporal domains which cover short-term and long-term variations. During the training, a context-dependent model is estimated according to the joint stylized $f_{0}$ contours over the syllable and a set of long-term temporal domains. During the synthesis, $f_{0}$ variations are determined using the long-term variations as trajectory constraints.
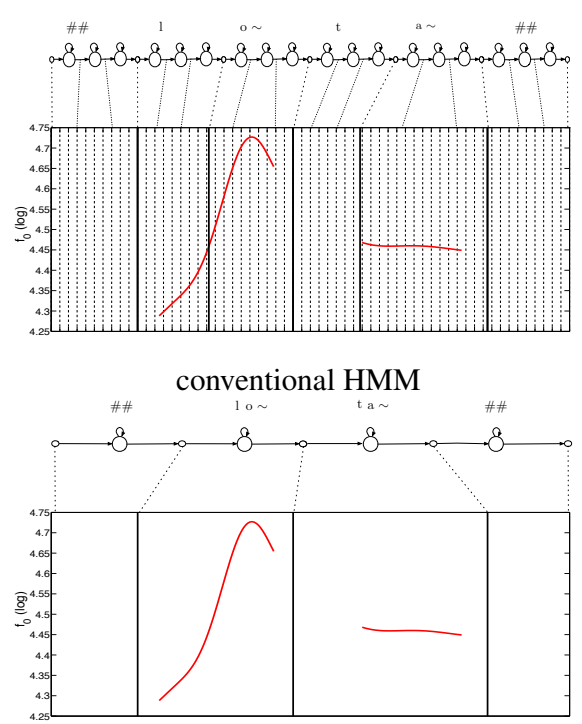

syllable-based HMM with stylization of $f_{0}$ contours

Figure 1: Schematic comparison of frame-based and syllablebased modelling of $f_{0}$ variations.

\section{Stylization of Speech Prosody}

The Discrete Cosine Transform (DCT) is used to stylize the $f_{0}$ variations over various temporal domains [11] (figure 2]. The 


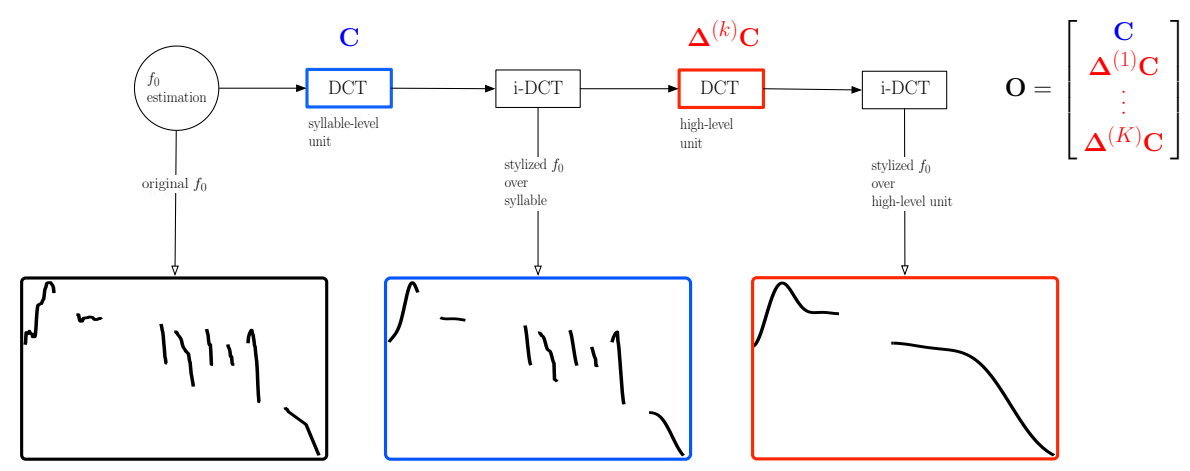

Figure 2: Instantaneous estimation of $f_{0}$, short-term stylization over syllable, and long-term stylization over prosodic group.

principle of the DCT is to decompose $f_{0}$ contours on a basis of slowly time-varying functions defined by zero-phase cosine functions $\phi=\left(\cos \left(\omega_{1}\right), \ldots, \cos \left(\omega_{T}\right)\right)$ at discrete frequencies $\omega_{k}=\frac{\pi}{2 T}(2 k+1)$, where $T$ is the length of the temporal domain used for stylization.

The stylized $f_{0}$ contour is then obtained by inverse transform of the $K$ order truncated DCT $(K \leq T)$ :

$$
f_{0}(t)=\sum_{k=1}^{K} \alpha_{k} c_{k} \cos \left(\omega_{k} t\right)
$$

where $c_{k}$ is the $k$-th term of the DCT, and $\alpha_{k}$ a term used for normalization.

Two classes of temporal domains are defined for the stylization of $f_{0}$ variations:

Syllable context accounts for $f_{0}$ variations occurring on the syllable and its immediate context (0-order represents the $f_{0}$ variations over the syllable, 1 -order the $f_{0}$ variations over the 1-left-to-right syllable context, ...);

Linguistic contexts account for $f_{0}$ variations occurring on long-term prosodic units (e.g., minor and major prosodic groups). A minor prosodic group is defined as the prosodic unit that ends with an intermediate prosodic boundary, and is used for rhythmic grouping typical of French. A major prosodic group is defined as the prosodic unit that ends with a major prosodic boundary.

$F_{0}$ variations are stylized using a 5-order DCT. $F_{0}$ is linearly interpolated in the logarithmic domain prior to the stylization. The stylization over various temporal scales aims at representing $f_{0}$ variations with more or less details, and to model short and long term dependencies.

\section{Trajectory Model}

The Trajectory Model has been introduced in HMM-based speech synthesis to explicitly model the dynamic (local variations) of the speech parameters [6]. In this study, syllable is assumed as the minimal temporal domain for the description of speech prosody, and $f_{0}$ variations are stylized and modelled simultaneously over different temporal domains: shortterm variations correspond to the stylization of $f_{0}$ contours over the syllable, and long-term variations correspond to the stylization of $f_{0}$ contours over long-term temporal domains. During the training, a context-dependent HMM is estimated from the joint short-term and long-term variations. During the synthesis, the short-term variations are determined so as to maximize the conditional probability of the short-term variations under the constraint of the long-term trajectories.

\subsection{Parameters Estimation}

Let $\mathbf{q}=\left[\mathbf{q}_{1}, \ldots, \mathbf{q}_{N}\right]$ be the sequence of linguistic contexts, where $\mathbf{q}_{n}=\left[q_{n}(1), \ldots, q_{n}(L)\right]^{\top}$ is a (Lx1) linguistic vector which describes the linguistic characteristics associated with the $n$-th syllable.

Let $\mathbf{c}=\left[\mathbf{c}_{1}, \ldots, \mathbf{c}_{N}\right]$ be the static observation sequence of stylized $f_{0}$ contours over the syllable-level unit, where $\mathbf{c}_{n}=$ $\left[c_{n}(1), \ldots, c_{n}(D)\right]^{\top}$ is a (Dx1) observation vector which describes the short-term $f_{0}$ characteristics associated with the $n$-th syllable.

Let $\boldsymbol{\Delta}^{(k)} \mathbf{c}=\left[\boldsymbol{\Delta}^{(k)} \mathbf{c}_{1}, \ldots, \boldsymbol{\Delta}^{(k)} \mathbf{c}_{N}\right]$ be the dynamic observation sequence of stylized $f_{0}$ contours over the $k$-th long-term temporal domain, where $\boldsymbol{\Delta}^{(k)} \mathbf{c}_{n}=\left[\Delta^{(k)} c_{n}(1), \ldots, \Delta^{(k)} c_{n}(D)\right]^{\top}$ is a (Dx1) observation vector which describes the long-term $f_{0}$ characteristics associated with the $n$-th syllable.

Let $\mathbf{o}=\left[\mathbf{o}_{1}, \ldots, \mathbf{o}_{N}\right]$ be the augmented observation sequence, where $\mathbf{o}_{n}=\left[\mathbf{c}_{n}^{\top}, \boldsymbol{\Delta}^{(1)} \mathbf{c}_{n}^{\top}, \ldots, \boldsymbol{\Delta}^{(K)} \mathbf{c}_{n}^{\top}\right]^{\top}$ is a $(\mathrm{KDx} 1)$ observation vector which describes the short-term and long term $f_{0}$ characteristics associated with the $n$-th syllable, and $K$ the total number of long-term temporal domains being modelled.

A HMM $\lambda_{\mathbf{q}}$ is estimated for each of the linguistic contexts. Each of the context-dependent HMMs is assumed to be a singlestate HMM with single normal distribution and diagonal covariance matrix. Then, a context-dependent HMM $\lambda$ is derived based on Maximum-Likelihood Minimum-Description-Length (ML-MDL). The long-term variations are used as additional trajectory constraints to refine the clustering of the models. A conventional context-dependent HMM is used to model syllable durations.

\subsection{Parameters Inference}

The determination of the sequence of $f_{0}$ parameters is similar to that of the Trajectory Model with the exception that the frame-based static observation is reformulated into the stylized $f_{0}$ contour over the syllable, and the frame-based dynamic observation (partial derivative) is reformulated into the stylized long-term $f_{0}$ contours. The sequence of syllable durations is determined with the conventional static method as the sequence of mean durations. 
The optimal static observation sequence $\mathbf{c}$ is determined so as to maximize the log-likelihood of the short-term observation sequence $\mathbf{o}$, under the constraint of the long-term trajectories $\Delta^{(\mathrm{k})} \mathbf{c}$

The optimal observation sequence $\widehat{\mathbf{o}}=\left[\widehat{\mathbf{o}}^{\top}, \ldots, \widehat{\mathbf{o}} T^{\top}\right]$ is determined so as to maximize the conditional probability of the observation sequence $\mathbf{o}$ given the model $\boldsymbol{\lambda}$.

$$
\widehat{\mathbf{o}}=\underset{\mathbf{o}}{\operatorname{argmax}} \max _{\mathbf{q}} \mathrm{p}(\mathbf{o} \mid \mathbf{q}, \boldsymbol{\lambda}) \mathrm{p}(\mathbf{q} \mid \boldsymbol{\lambda})
$$

The determination of the optimal observation sequence o divides into the following sub-problems:

$$
\begin{aligned}
& \widehat{\mathbf{q}}=\underset{\mathbf{q}}{\operatorname{argmax}} \mathrm{p}(\mathbf{q} \mid \boldsymbol{\lambda}) \\
& \widehat{\mathbf{o}}=\underset{\mathbf{o}}{\operatorname{argmax}} \mathrm{p}(\mathbf{o} \mid \widehat{\mathbf{q}}, \boldsymbol{\lambda})
\end{aligned}
$$

Assuming that each syllable is modelled by a single-state HMM, the optimal state sequence simply corresponds to the concatenated sequence of context-dependent models associated with each syllable of the syllable sequence:

$$
\widehat{\mathbf{q}}=\left[\mathbf{q}_{1}, \ldots, \mathbf{q}_{N}\right]
$$

where $N$ denotes is the total number of syllables in the syllable sequence.

The maximization of $p(\mathbf{o} \mid \widehat{\mathbf{q}}, \boldsymbol{\lambda})$ with respect to $\mathbf{o}$ is equivalent to the maximization of $\mathrm{p}(\mathbf{c} \mid \widehat{\mathbf{q}}, \boldsymbol{\lambda})$ with respect to $\mathbf{c}$ under the dynamic constraints $\boldsymbol{\Delta}^{(k)} \mathbf{c}$ :

$\widehat{\mathbf{o}}=\underset{\mathbf{o}}{\operatorname{argmax}} \mathrm{p}(\mathbf{o} \mid \widehat{\mathbf{q}}, \boldsymbol{\lambda}) \Leftrightarrow \widehat{\mathbf{c}}=\underset{\mathbf{c}}{\operatorname{argmax}} \mathrm{p}(\mathcal{F}(\mathbf{c}) \mid \widehat{\mathbf{q}}, \boldsymbol{\lambda})$

under the constraint:

$$
\mathbf{o}=\mathcal{F}(\mathbf{c})=\left[\mathbf{c}^{\top}, \boldsymbol{\Delta}^{(\mathbf{k})} \mathbf{c}^{\top}, \ldots, \boldsymbol{\Delta}^{(\mathbf{K})} \mathbf{c}^{\top}\right]^{\top}
$$

A local solution to this problem is determined recursively using a quasi-Newton method. Finally, global variance is used to model global dynamics [7].

\section{Evaluation}

\subsection{Stimuli}

The proposed trajectory model was evaluated and compared to the conventional HMM-based model in a subjective evaluation in speech synthesis. Four models were compared: 1) the conventional HMM-based model (HTS), and trajectory models using different long-term temporal domains: 2) syllable +1 -order syllable-context (1ORDER), 3) syllable + minor prosodic group $(\mathrm{AG})$, and 4) syllable + major prosodic group (PG). Evaluation was conducted using the HMM-based speech synthesis system [1]. Models were trained on 5 hours (1888 utterances) of a French single-speaker story-telling speech database using conventional linguistic contexts. 8 sentences randomly extracted from the fairy-tale "Le Petit Poucet" ("Little Tom Thumb") were used for the comparison. For each of the trajectory models, the inferred sequence of stylized $f_{0}$ parameters was converted into a sequence of $f_{0}$ variations with respect to the inferred syllable durations and the voice/unvoiced sequence as inferred from the conventional HMM-based $f_{0}$ model. Finally, speech utterances were synthesized by the speech synthesizer. Each sentence was synthesized with the different models.

\subsection{Procedure}

20 native French speakers (including 13 expert and 7 naïve listeners) participated in the evaluation. The experiment consisted in a subjective comparison of the different speech prosody models. A comparison category rating test was used to compare the naturalness of the synthesized speech utterances. The evaluation was conducted according to a crowd-sourcing technique using social networks. Pairs of synthesized speech utterances were randomly presented to the participants. They were asked to attribute a preference score according to the naturalness of the speech utterances being compared on the comparison mean opinion score (CMOS) scale.

\section{Results}

Overall CMOS and preference score (PS) are presented in figure 3 The 1-order trajectory model significantly outperforms all of
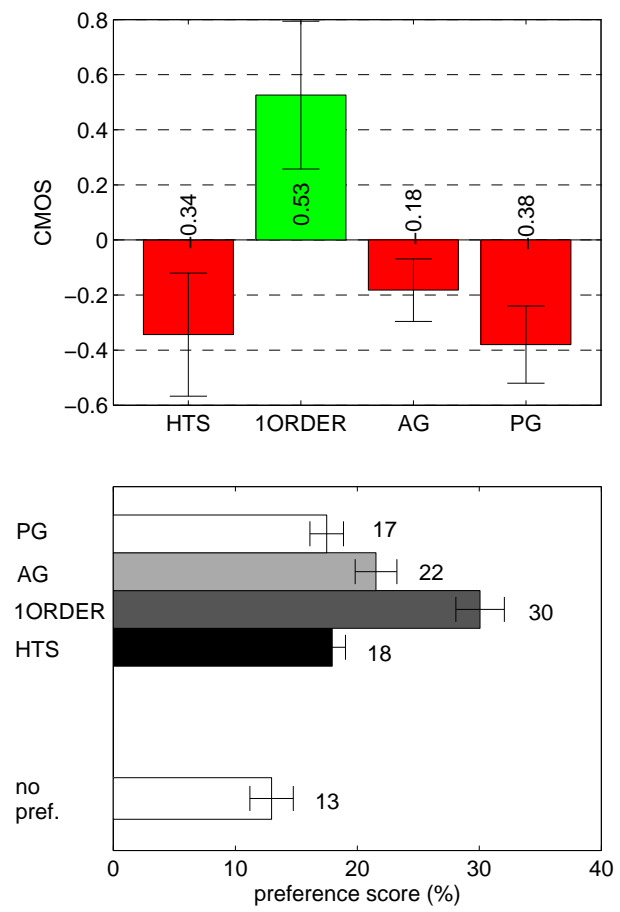

Figure 3: CMOS and PS. Mean and 95\% confidence intervals

the other prosodic models whatever is the preference measure. In particular, the 1-order trajectory model is overally significantly preferred to the other prosodic models $(\mathrm{CMOS}=+0.53$, $\mathrm{PS}=30 \%$ ), and is individually significantly preferred to each of the other prosodic models $(\mathrm{MOS}=+0.54,+0.51,+0.54$ and PS $=52.1 \%, 56.3 \%, 55.1 \%$ compared with HTS, AG, and PG models respectively). The AG trajectory model is preferred to the HTS model but not significantly (overall: $\mathrm{CMOS}=-0.18$, $\mathrm{PS}=22 \%$; pair: $\mathrm{CMOS}=+0.15$, $\mathrm{PS}=46 \%$ ); and significantly preferred to the PG trajectory model. Finally, the HTS model is preferred to the PG trajectory model, but not significantly (overall: $\mathrm{CMOS}=-0.34, \mathrm{PS}=18 \%$; pair: $\mathrm{CMOS}=+0.10, \mathrm{PS}=28.7 \%$ ). In particular, trajectory models decrease in preference when increasing the temporal domain of the trajectory constraint $\left(\mathrm{CMOS}_{1-\text { order }}=+0.53, \mathrm{PS}_{1-\text { order }}=30 \% ; \quad \mathrm{CMOS}_{\mathrm{AG}}=-0.18\right.$, $\left.\mathrm{PS}_{\mathrm{AG}}=22 \% ; \mathrm{CMOS}_{\mathrm{PG}}=-0.38, \mathrm{PS}_{\mathrm{PG}}=17 \%\right)$. 
A comparison of the preference scores depending on the expertise of the participant reveals a significant difference in the perception of speech prosody between naïve and expert listeners : naïve listeners have clearly marked preferences, but with more variability, while expert listeners have less marked preferences, but with less variability (table 1 ).

\begin{tabular}{l|cc|c|cc|c}
\multirow{2}{*}{ CMOS } & \multicolumn{3}{|c|}{ naive } & \multicolumn{3}{c}{ expert } \\
\cline { 2 - 7 } & \multicolumn{2}{|c}{ score } & rank & \multicolumn{2}{|c}{ score } & rank \\
\hline HTS & -0.77 & $( \pm 0.44)$ & 4 & -0.20 & $( \pm 0.27)$ & 2 \\
1-order & $\mathbf{+ 0 . 8 8}$ & $( \pm 0.43)$ & $\mathbf{1}$ & $\mathbf{+ 0 . 4 1}$ & $( \pm 0.26)$ & $\mathbf{1}$ \\
AG & -0.10 & $( \pm 0.50)$ & 2 & -0.21 & $( \pm 0.28)$ & 3 \\
PG & -0.20 & $( \pm 0.44)$ & 3 & -0.52 & $( \pm 0.24)$ & 4
\end{tabular}

Table 1: CMOS depending on the expertise of the participant. Mean score and $95 \%$ confidence interval.

\section{Discussion}

A study case of synthesized $f_{0}$ variations with respect to the speech prosody model is provided in figure 4 with prior state duration alignment. Speech prosody differences mostly concern $f_{0}$ variations, and no significant differences between state-based and syllable-based modelling.

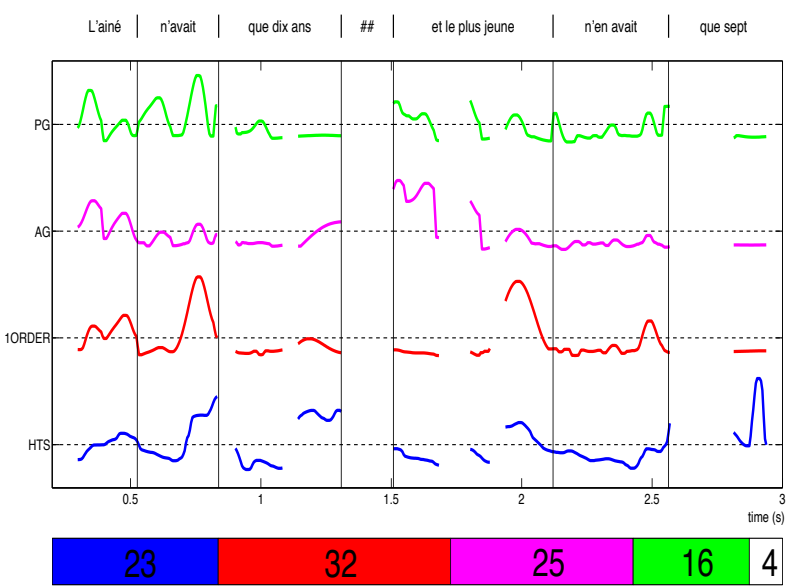

Figure 4: Comparison of synthesized $f_{0}$, with PSs.

The 1-order trajectory model clearly succeeds to model the local variations and dynamic of speech prosody. The synthesized $f_{0}$ variations presents an expanded dynamics while less micro-prosodic details than those synthesized by the HTS model. Thus, naïve listeners may focus on global variations only, when expert listeners may pay a closer attention to finer prosodic details. The AG trajectory model appears to model middle-term prosodic variations such as initial $f_{0}$ reset and local $f_{0}$ declination, compared with the 1-order trajectory model and the HTS model. However, dynamics is less expended, and prosodic phrasing is more flat.

A comparison of the different trajectory models reveals that differences in speech prosody concern local (syllable contours and dynamics) and global $f_{0}$ variations. However, it is observed that the increase of the trajectory domain results into noisy local $f_{0}$ variations, and partially (AG) or totally (PG) inadequate global $f_{0}$ contours. In particular, the PG trajectory model failed in modelling global $f_{0}$ declination. The degradation is probably due to the increase in the dimensionality of the optimization problem when accounting for long-term trajectory constraints. In the absence of an explicit formulation of the gradient, the optimization method obviously failed to account for the long-term dependencies. Not surprisingly, this results both into local and global degradation in the synthesized $f_{0}$ variations.

\section{Conclusion}

In this paper, a trajectory model based on the stylization and the joint modelling of $f_{0}$ variations over various temporal domains was proposed. In the proposed approach, $f_{0}$ variations are stylized with a Discrete Cosine Transform, and modelled simultaneously over various temporal domains which cover short-term and long-term variations. During the training, a context-dependent model is estimated according to the joint stylized $f_{0}$ contours over the syllable and a set of long-term temporal domains. During the synthesis, $f_{0}$ variations are inferred using the long-term variations as trajectory constraints. The evaluation consisted in a subjective comparison of different speech prosody models in speech synthesis.

The 1-order trajectory model was proved to be significantly preferred to the conventional model, and to the other trajectory models. Each of the trajectory models succeeds in modelling $f_{0}$ contours that are consistent with the considered temporal domains. However, the ability of the trajectory model to account for long-term variations decreases when the temporal domain increases, due to the increase in complexity of the optimization process. In further studies, the relationship between static and dynamic trajectories will be explicitly formulated, and different combinations of trajectory constraints will be evaluated. Finally, the formulation of the trajectory model will be extend to the modelling of the local speech rate variations.

\section{References}

[1] H. Zen, K. Tokuda, and A. Black, "Statistical parametric speech synthesis," Speech Communication, vol. 51, no. 11, pp. 1039-1064, 2009.

[2] H. Fujisaki, The Production of Speech. Springer, New York, 1983, ch. Dynamic characteristics of voice fundamental frequency in speech and singing, pp. 39-55.

[3] J. Van Santen and B. Moebius, Intonation Analysis, Modelling and Technology. Kluwer Academic, Netherlands, 1999, ch. A quantitative model of f0 generation and alignment, pp. 269-288.

[4] T. Yoshimura, K. Tokuda, T. Masuko, T. Kobayashi, and T. Kitamura, "Simultaneous modeling of spectrum, pitch and duration in HMM-based speech synthesis," in European Conference on Speech Communication and Technology, Budapest, Hungary, 1999, pp. 2347-2350.

[5] H. Zen, K. Tokuda, T. Masuko, T. Kobayashi, and T. Kitamura, "Hidden semi-Markov model based speech synthesis," in International Conference on Speech and Language Processing, Jeju Island, Korea, 2004, pp. 1397 1400

[6] K. Tokuda, H. Zen, and T. Kitamura, "Trajectory modeling based on HMMs with the explicit relationship between static and dynamic features," in $E u$ ropean Conference on Speech Communication and Technology, Geneva, Switzerland, 2003, pp. 865-868.

[7] T. Toda and K. Tokuda, "A speech parameter generation algorithm considering global variance for HMM-based speech synthesis," IEICE Transactions on Information and Systems, vol. 90, no. 5, pp. 816-824, 2007.

[8] J. Latorre and M. Akamine, "Multilevel parametric-base F0 model for speech synthesis," in Interspeech, Brisbane, Australia, 2008, pp. 22742277.

[9] Y. Qian, Z. Wu, and F. K. Soong, "Improved prosody generation by maximizing joint likelihood of state and longer units," in International Conference on Acoustics, Speech and Signal Processing, Taipei, Taiwan, 2009, pp. 3781-3784.

[10] B. Gao, Y. Qian, Z. Wu, and F. Soong, "Duration refinement by jointly optimizing state and longer unit likelihood," in Interspeech, Brisbane, Australia, 2008 , pp. 2266-2269.

[11] J. Teutenberg, C. Watson, and P. Riddle, "Modelling and Synthesising F0 contours with the Discrete Cosine Transform," in International Conference on Acoustics, Speech, and Signal Processing, Las Vegas, U.S.A, 2008, pp. 3973-3976. 